\title{
Computing Inspiration: i.plot
}

\author{
Naoko Tosa', \\ Seigow Matsuoka ${ }^{2}$, and Ryohei Nakatsu ${ }^{3}$ \\ ${ }^{1}$ Kyoto Univeristy, Academic Center for Computing and Media Studies, \\ Yoshida-Nihon-Matsu, Sakyo, 606-8501 Kyoto, Japan \\ tosa@media.kyoto-u.ac.jp \\ ${ }^{2}$ Editorial Engineering Laboratory, 7-6-64 Akasaka Minato-ku \\ 107-0052, Tokyo, Japan \\ ${ }^{3}$ National University of Singapore, Interactive Digital Media Institute \\ 4 Engineering Drive 3, Singapore 117576 \\ idmdir@nus.edu.sg
}

\begin{abstract}
In this paper we treat the theme of "the future of narrative." In examining how a computer can inspire with humor and wisdom, we studied the hidden relationships and contextual emergence of language. Pursuing a vision of the future where people will have conversations with robots, we not only display the results visually, but also have a robot agent convey inspiration and emotional content to users.
\end{abstract}

\section{Introduction}

Narrative is at its most vivid when emergent technologies are born. "Emergent" means when a product or idea, in the course of its advancement, breaks through a critical barrier, and a heretofore-unimagined paradigm appears. It makes sense that this kind of occurrence can lead to the discovery of new relationships and the creation of fresh images. The trick to finding this kind of emergence is daring to pursue the marriage of completely different ideas.

Since it is expected that the convergence of art and technology will lead society, much thought has been put into the intersection of these two fields. In the history of art and technology, which began in the late 1960's, the one thing that has not been researched enough is the field's relationship to literary narrative. This is perhaps because, due to technological factors, those in the field of literature were not able to approach the interdisciplinary area of art and science. But now, with computers as an inter-medium finally beginning to mature, it has become possible for men and women in the field of literature to get closer to computers. 
Thus, with "the future of narrative" as a theme, we researched how a computer can offer humor, wisdom and inspiration to a user. Up until now, topics researched within art and science have fallen mainly under the category of nonverbal communication, such as feeling and atmosphere. With our research, the logical meaning of language and its intuitive aspects such as atmosphere and feeling have joined together. We can promise with confidence that in the future, through the addition of narrative technology to art and science, a new, creative "interactive narrativity" will develop, and its developing value will be a significant contribution to the field of art and technology.

\section{Inspiration Generation}

With traditional context generation and search, words close contextually to a word are chosen by searching through a regular thesaurus. However, with this kind of technique, it is very difficult to produce an interesting context from inspiration will rise out. What is the essence of inspiration? According to the dictionary, it is a new idea that flashes within the creative and speculative process - an idea that did not previously exist in the thinker's mind.

We have realized a method of emergent context generation called Inspiration Computing and developed a system called i.plot. The key feature of i.plot is a system that discovers hidden connections between unrelated words by tracing possible paths through the database. The path between words is like that of a wandering mind, where the jump between each idea is clear but multiple jumps lead to unexpected results. The user may highlight interesting sets of words and delve deeper into the word associations. Other features of i.plot are described below.

\section{Technical Realization}

The i.plot system is built using open-source software on a Linux platform, and it is accessible via the World Wide Web. The system takes advantage of several publicly available resources and a chaos-based algorithm to produce its inspiration output, using a robot agent to interpret the results.

\subsection{Database}

With traditional context generation, words close contextually to a word are chosen by searching through a regular thesaurus. However, with this technique, it is difficult to produce interesting results. We have realized a method of emergent context 
generation we call Inspiration Generation. This method uses a combination of the WordNet lexical database, the Edinburgh Psychological Associative Thesaurus, and Seigow Matsuoka's original "thoughtforms" to produce a dynamic working base with a vocabulary of over 20,000 words.

\subsection{Thoughtforms}

"Thoughtforms," developed by our collaborator Seigow Matsuoka, are forms for the editing of words. They comprise the following five types:

Concatenation. This is information of the same source group arranged in order of continuity, stratum or ranking, such that the "space" between the separate words matches up. For example, "Top," "middle" and "bottom": they are all words that specify position; they are in a ranked order; and there are no discontinuous jumps between the words.

\section{Concatenation}

\section{Egg - Chick - Hen}

Fig. 1. Concatenation thoughtform example

Balance. Just like "reading, writing and arithmetic" or "food, clothing and shelter," there are many examples of three-sets of words that we use frequently. It is good to find an equally balanced set such that the words push and pull on one another with the same amount of force.

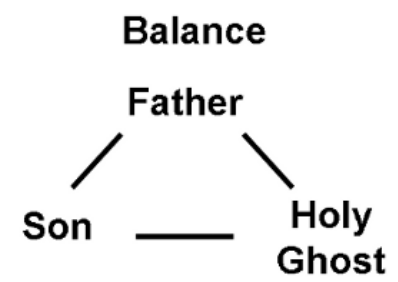

Fig. 2. Balance thoughtform example 
Division. This is the division of an idea into two child ideas. Depending on which aspect of the parent we take, the child ideas become separate but complete ideas. So, if we split "computer" into two child ideas, we produce two elements indispensable to a computer.

\section{Division}

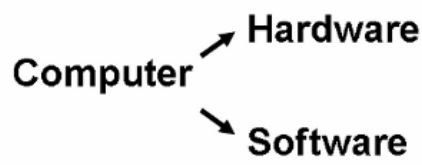

Fig. 3. Division thoughtform example

Unification. A combination of two ideas to produce a new idea. This is not just a simple addition of ideas. This is the creation of a new word or image - a new paradigm - through the combination and synthesis of two ideas. It is also not just a simple reversal of the "division" pattern. This "unification" pattern comes in very handy when trying to develop new products or concepts, or in naming a new idea.

\section{Unification}

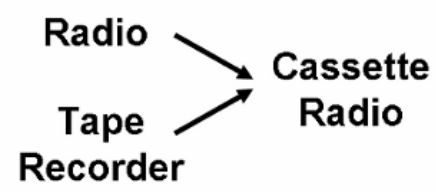

Fig. 4. Unification thoughtform example

Crisscross. This is a pattern in which four ideas are derived from a central idea. There are two types of Crisscross patterns: (1) where the outlying ideas are composed in parallel - one could think of this as a four-point version of the "balance" pattern - and (2) where the ideas are composed as separate axes, creating a grid as below, from which we see ideas such as "old man" and "young woman." 


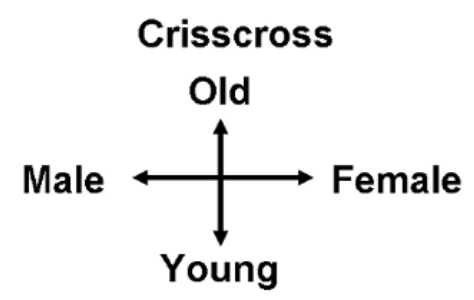

Fig. 5. Crisscross thoughtform example

\subsection{Chaos Search}

The system uses a dual-synchronized chaos engine, which synchronizes two or more chaos states, to increase the variety of idea-word connections. Each time a user refreshes the display, the entropy of the chaos engine increases. The engine, which runs continuously in the background, contains an Objective chaos, a User chaos following the Objective, and a System value controlling the synchronization of User and Objective. By increasing the System value, the engine produces more chaotic output, and connection lengths vary from short and direct to long and round-about.

\subsection{Inspiration Space}

This system discovers the hidden connections between words, defining the basic function for each of the other systems.

We collected a database of thoughtform relationships created by students in Japan and added connection data from the Edinburgh Associative Thesaurus (Kiss, et al.), which contains stimulus-response data from a large sample group of people. We define a connection between words to exist if two words are found in the same thoughtform or make up a stimulus-response pair in the associative thesaurus.

The system finds several connections between two unrelated words by tracing a large set of possible paths between the two words, such that the paths traverse several two-word connections. If the chaos engine is in an appropriate state, a preference may be added so that longer paths are displayed, or so that the paths are forced to connection through a more distantly-connected word. The user may further expand the connections of any word of interest. 


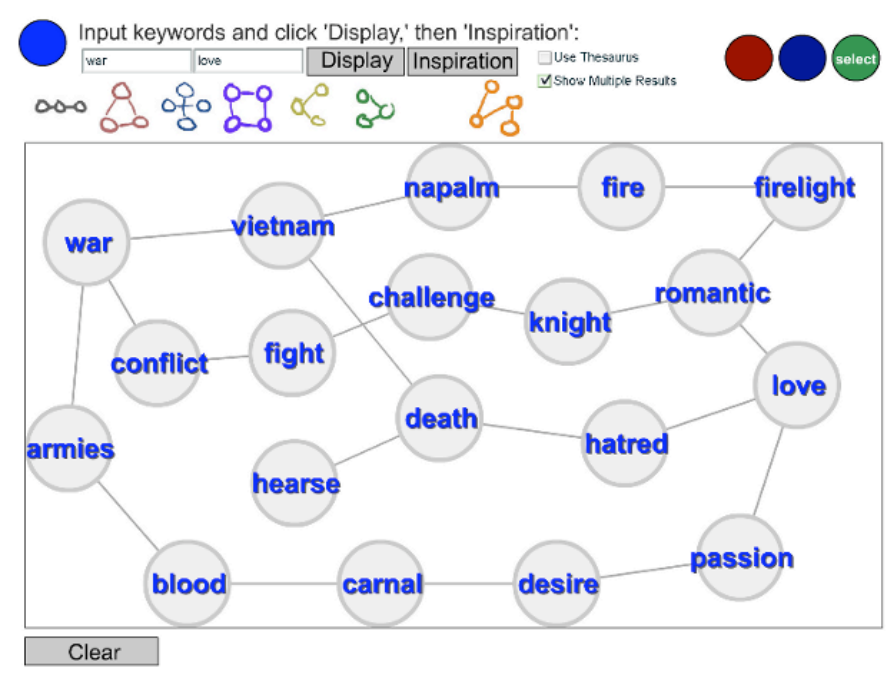

Fig. 6. An example result from Inspiration Space

\section{Possible Applications}

\subsection{Inspiration Restaurant Guide}

We used information from France Telecom's Yellow Pages database to build a restaurant guide based on our inspiration system.

We entered each of the restaurant categories and locations in Paris into our system, and connected them to related words (i.e. Pizzeria: italian food, tomato; Creperie: date, sweets; Fast Food: quick, cheap). Thus we were able to build inspirational links to each of the restaurant types and physical locations.

The user selects a location (or lets the system choose for him), inputs their preferences for restaurant atmosphere, and a set of words appears. The user can select any word that appears on the screen, and the system searches for a restaurant type closely related to that word. A nearby restaurant of that type is then displayed on the screen. 


\section{Inspiration Restaurant Guide}

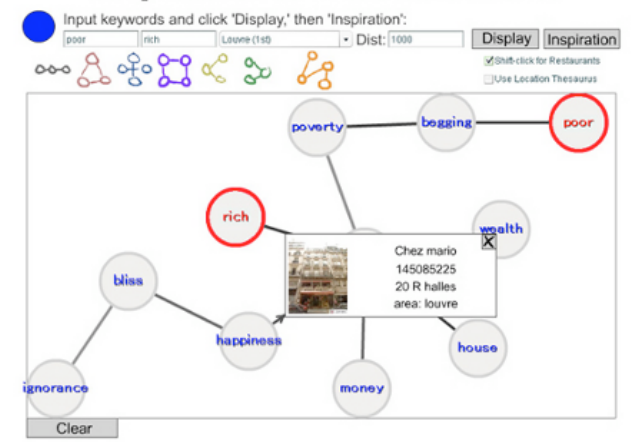

Fig. 7. Example result from Inspiration Restaurant Guide

\subsection{Context Inspiration}

Using data obtained from the open database WordNet (Princeton University) as well as manual categorization, we classified the words in our database according to their grammatical properties, fitting six categories: who, what, where, when, how, and verb.

The user seeds the system with a few idea words. The system then generates a sentence of various lengths ranging from 2 to 5 words (minus articles, conjunctions, etc.) based on these input words. Wherever there is a blank word, the system fills it in, seeking words inspirationally linked to the words surrounding a blank in the sentence. In the case that there is a word on either side of the blank, we use the same algorithm as for finding a connection between words in the Inspiration Space program, except we look for an intersection point that is of an appropriate grammatical type to fit in the sentence. 


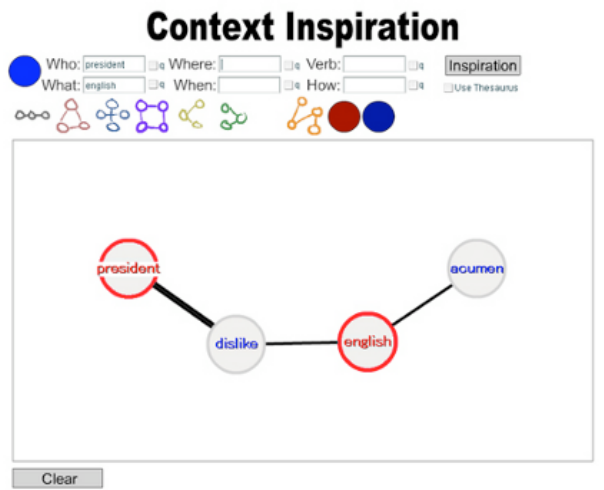

Fig. 8. Example result from Context Inspiration

\subsection{Symbol Inspiration}

Rather than attaching symbol images onto existing word associations, we created a set of associations directly between symbols. These associations are based on the thoughtforms explained above, where connections are based on geometric forms.

One can either seed the engine by entering in words linked to images in the input textboxes, or by clicking one of the colored thoughtform buttons at the top of the screen. One can select various images to expand the relationships.

By inserting an element of unpredictability from the chaos engine, the connections between images may shift and change, avoiding the draw of logic.

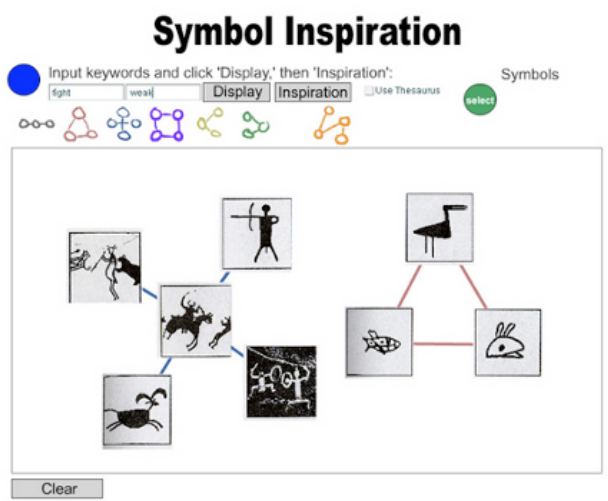

Fig. 9. Example result from Symbol Inspiration 


\subsection{Inspiration Blog}

The blog system adds the ability to take complete sentences as input. Connections between key words in the sentence are all considered, and intersecting words are displayed on the screen. The connections between each entry and the preceding entry are also included, so that the context generated within each entry is continuous.

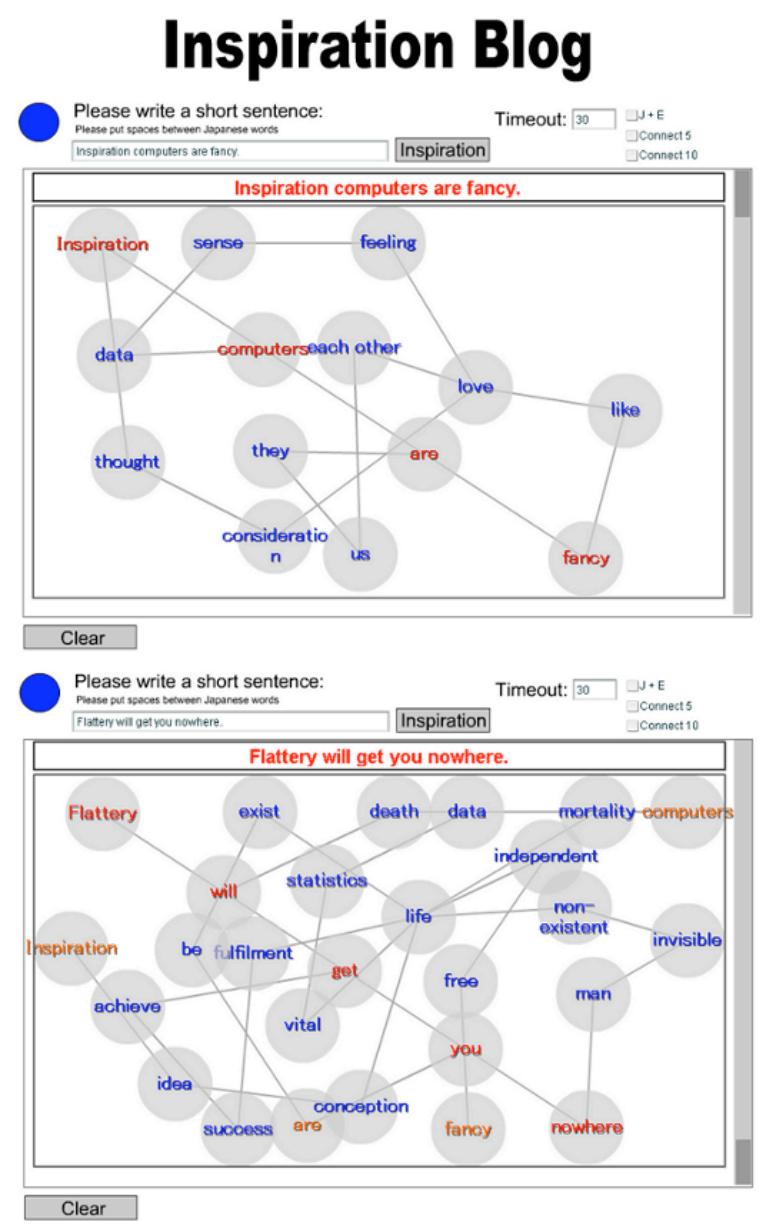

Fig. 10. Example result from Inspiration Blog 


\section{Robot Agent}

Pursuing a vision of the future where people will have conversations with robots, we not only display the results visually, but also have a robot agent convey inspiration and emotional content to users. The robot takes the output and delivers it with behaviors, tai-chi motions and emotional voice synthesis appropriate to the inspiration words. We connected a text-to-speech synthesis engine with the robot, enabling it to perform emotional behaviors and graceful motions in communication with humans. When a user interacts with the inspiration system, the key words are extracted by the system and converted into behaviors via a languageemotion mapping, which the system sends to the robot to perform.

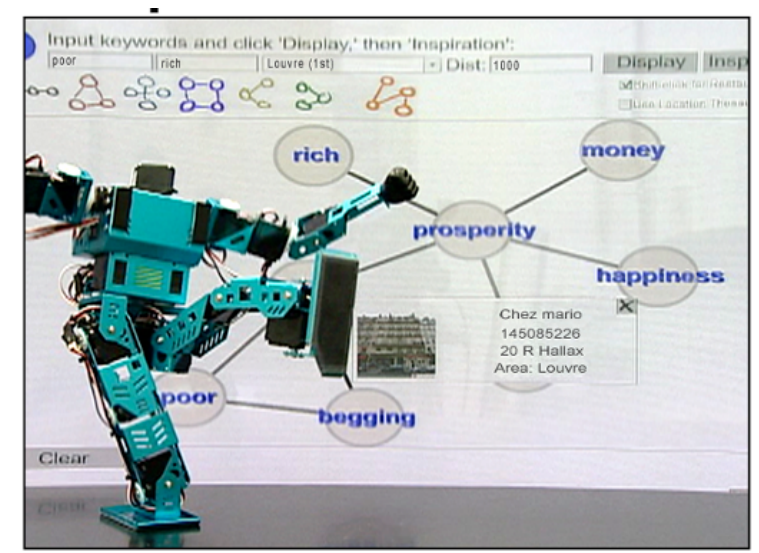

Fig. 11. The robot agent presenting a result from Inspiration Restaurant Guide

\section{Future work}

Among traditional methods for teaching an idea and passing it on, there exists the method of conveying information via story. The next thing that will become necessary for the interaction technology and expression between computers and humans is research in interactive narrative methods. Our research will likely have a significant impact in this up-and-coming field of interactive narrative methods.

Among the human computer interaction within our daily lives, there are still many logical, simple and boring interactions. Our research, in providing users with inspiration containing humor and wisdom, offers people new opportunities for stimulation; our future symbiosis with computers as well as digital contents (education, games and entertainment) will therefore change significantly, in that 
computers and robots will convey to people the relationships, breadth of meaning and context hidden between words not easily struck upon by people.

\section{References}

1. Janet H. Murray, "Hamlet on the Holodeck".

2. Bela Balazs, "Film theory".

2. Joe Bates, et al. "Oz Project".

3. WordNet (Princeton University, http://www.cogsci.princeton.edu/ wn/)

4. Edinburgh Associative Thesaurus (Kiss, et al.; http://www.eat.rl.ac.uk/) 